\title{
On Finding Spherical Geodesic Paths and Circles in $\mathbb{Z}^{3}$
}

\author{
Ranita Biswas and Partha Bhowmick \\ Department of Computer Science and Engineering \\ Indian Institute of Technology, Kharagpur, India \\ \{biswas.ranita, bhowmick\}@gmail.com
}

\begin{abstract}
A discrete spherical geodesic path between two voxels $s$ and $t$ lying on a discrete sphere is a/the 1-connected shortest path from $s$ to $t$, comprising voxels of the discrete sphere intersected by the real plane passing through $s, t$, and the center of the sphere. We show that the set of sphere voxels intersected by the aforesaid real plane always contains a 1-connected cycle passing through $s$ and $t$, and each voxel in this set lies within an isothetic distance of $\frac{3}{2}$ from the concerned plane. Hence, to compute the path, the algorithm starts from $s$, and iteratively computes each voxel $p$ of the path from the predecessor of $p$. A novel number-theoretic property and the 48-symmetry of discrete sphere are used for searching the 1-connected voxels comprising the path. The algorithm is output-sensitive, having its time and space complexities both linear in the length of the path. It can be extended for constructing 1connected discrete 3D circles of arbitrary orientations, specified by a few appropriate input parameters. Experimental results and related analysis demonstrate its efficiency and versatility.
\end{abstract}

Keywords: Discrete sphere, geodesic path, geometry of numbers, discrete 3D circles.

\section{Introduction}

The shortest path between two points on a curved surface is called geodesic. There exist several works related to geodesics on a 3D triangulated surface, e.g., the fast marching technique [8]. This technique and Polthier's straightest geodesics theory [13] are used in [11] for finding approximate geodesics on triangulated surfaces. For exact geodesics, a cubic-time line-of-sight algorithm is proposed in [1].

The first algorithm to solve the discrete geodesic problem as the shortest path (SP) between a source and a destination point on an arbitrarily polyhedral surface is referred in the literature as MMP 12 . The discrete surface points are first preprocessed and stored in a suitable data structure in $O\left(n^{2} \log n\right)$ time, and then the actual SP is reported by continuous Dijkstra's algorithm in $O(k+\log n)$ time, where $n=$ \#edges on the surface and $k=$ \#faces crossed by SP. Improving MMP to $O\left(n^{2}\right)$ time complexity is done in $\mathrm{CH}$ algorithm [4 using a set of 
windows on the polyhedron edges for encoding the shortest paths. However, it is shown in 14 that MMP, in practice, runs faster than CH. Later, it has been shown in [16] that $\mathrm{CH}$ can be made to run faster than MMP, using priority queue and filtering out the useless windows. Recently, a parallel version of $\mathrm{CH}$ is proposed in [19]. Further developments with graph-theoretic and numerical methodologies may be seen in [17, 18].

Problems related to geodesic paths and their characterization in the digital space have gained significant attention in recent time. In [5], a new geodesic metric and the $A^{*}$ algorithm are used to find the shortest path between a source and a destination voxel. In [3], rubberband algorithm is proposed for computation of minimum-length polygonal curves in cube-curves in 3D space. The idea can be extended to solve various Euclidean shortest path (ESP) problems inside of a simple cube arc, inside of a simple polygon, on the surface of a convex polytope, or inside of a simply-connected polyhedron [10.

In $\mathbb{R}^{3}$, a spherical geodesic path is defined between two points $p \in \mathbb{R}^{3}$ and $q \in \mathbb{R}^{3}$ lying on a real sphere $S_{r}^{\mathbb{R}}$ of radius $r$. The path always lies along the intersection circle of $S_{r}^{\mathbb{R}}$ and the 3D plane passing through $p, q$, and the center of $S_{r}^{\mathbb{R}}$. We make an analogous definition for discrete spherical geodesic path $\boldsymbol{\pi}_{r}^{\mathbb{Z}}(s, t)$ from a point (voxel) $s \in \mathbb{Z}^{3}$ to another point $t \in \mathbb{Z}^{3}$ lying on the discrete sphere, $S_{r}^{\mathbb{Z}}$, of radius $r$. W.l.o.g., we fix the center of $S_{r}^{\mathbb{Z}}$ at $o(0,0,0)$, and consider $r$ as a positive integer. Then, $\boldsymbol{\pi}_{r}^{\mathbb{Z}}(s, t)$ is defined as a/the 1-connected shortest path from $s$ to $t$, comprising only those voxels of $S_{r}^{\mathbb{Z}}$ which lie sufficiently close to the real plane $\Pi_{r}^{\mathbb{R}}(s, t)$ passing through $s, t$, and $o$.

We first show that there always exists a 1-connected cycle in the set $I_{r}^{\mathbb{Z}}(s, t)$ comprising the voxels of $S_{r}^{\mathbb{Z}}$ intersected by $\Pi_{r}^{\mathbb{R}}(s, t)$. The set $I_{r}^{\mathbb{Z}}(s, t)$ admits the characterization that all its voxels lie within an isothetic distance of $\frac{3}{2}$ from $\Pi_{r}^{\mathbb{R}}(s, t)$. Subsequently, $\boldsymbol{\pi}_{r}^{\mathbb{Z}}(s, t)$ becomes a subset of $I_{r}^{\mathbb{Z}}(s, t)$, and is efficiently obtained by a prioritized Breadth-First-Search algorithm on the underlying graph corresponding to $I_{r}^{\mathbb{Z}}(s, t)$. For computation of $I_{r}^{\mathbb{Z}}(s, t), S_{r}^{\mathbb{Z}}$ is defined as the irreducible 2-separable set of voxels (3D integer points) that are uniquely identified by certain number-theoretic properties. The algorithm computes the set $I_{r}^{\mathbb{Z}}(s, t)$ using these properties, without considering the entire set $S_{r}^{\mathbb{Z}}$. Figure 1 shows a result of our algorithm, where the search space of BFS, its 18 neighborhood on $S_{r}^{\mathbb{Z}}$, and the final geodesic path $\boldsymbol{\pi}_{r}^{\mathbb{Z}}(s, t)$ are shown in different colors.

The rest of the paper is organized as follows. Section 2 explains certain elementary number-theoretic properties of a digital sphere, used for computing $I_{r}^{\mathbb{Z}}(s, t)$. Section 3 contains characterization of discrete spherical geodesic path and circle. The algorithm to compute the geodesic path from a point $s$ to a point $t$ lying on $S_{r}^{\mathbb{Z}}$ is presented in Section 4 . Section 5 contains some test results, and Section 6 the concluding notes.

\section{Digital Sphere}

We first introduce definitions and properties of digital sphere related to this work. These are subsequently used to design the algorithms for finding geodesic 

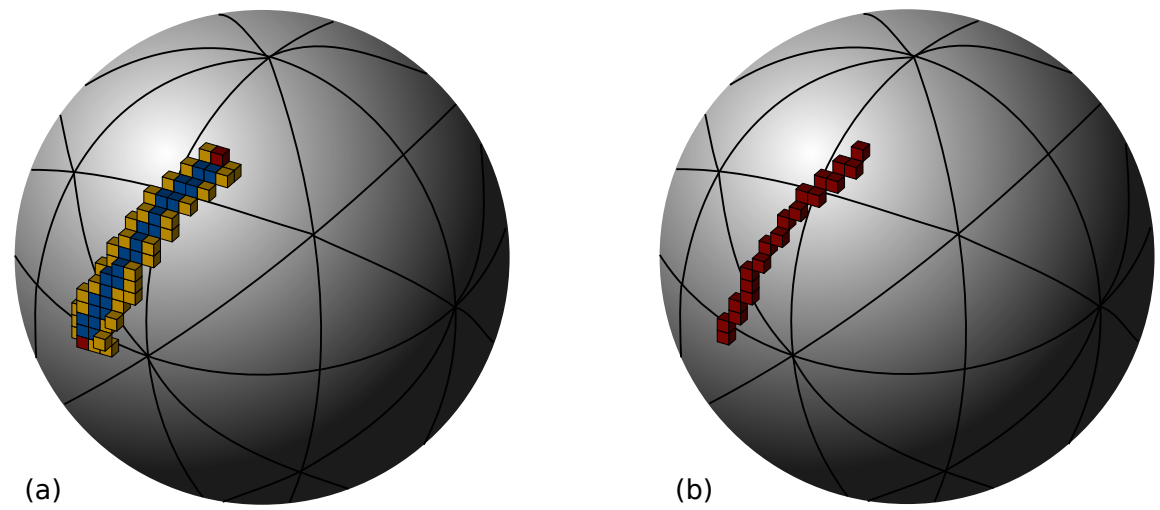

Fig. 1. A geodesic path reported by the proposed algorithm for $r=17$. (a) Red: $s(-6,-1,16)$ and $t(2,14,10)$; blue: $I_{r}^{\mathbb{Z}}(s, t)$; yellow: 18-neighborhood of the breadthfirst search space. (b) The geodesic path $\boldsymbol{\pi}_{r}^{\mathbb{Z}}(s, t)$ shown in red.

paths and 3D circles in $\mathbb{Z}^{3}$. The first point to observe is that, opposed to a real sphere, a digital sphere has only nine planes of symmetry. Three of these are the planes containing the great circles parallel to three coordinate planes; and for each of these three planes, there exist two more planes aligned at $+45^{0}$ and $-45^{0}$ to it. These nine planes of symmetry give rise to eight coordinate octants, called c-octants. Each c-octant contains 6 Möbius triangles [7, thus dividing the sphere into 48 quadraginta octants or q-octants.

\subsection{Representation}

The c-octants and the q-octants are uniquely represented by 3-tuples (see Appendix), which are carefully prepared for efficient implementation of our algorithm. Each c-octant $\mathbb{C}_{i}$ is represented by a 3 -tuple of signs of coordinate axes, namely $C_{i}:=\left(c_{i}^{(1)}, c_{i}^{(2)}, c_{i}^{(3)}\right)$. For example, $C_{1}=(+,+,+), C_{2}=(-,+,+)$, and so forth. The 3 -tuple for each q-octant, on the contrary, represents the three signed coordinate axes. In particular, in the 3-tuple $Q_{i}:=\left(q_{i}^{(1)}, q_{i}^{(2)}, q_{i}^{(3)}\right)$ representing $\mathbb{Q}_{i}$, each element $q_{i}^{(\cdot)}$ has two variables, namely $\omega$ and $\sigma$. The variable $\omega$ contains a literal (name of the coordinate axis) from $\{\mathrm{x}, \mathrm{y}, \mathrm{z}\}$, and the variable $\sigma$ contains the sign of the corresponding coordinate. With this representation, we have $Q_{1}=(+\mathrm{x},+\mathrm{y},+\mathrm{z}), Q_{2}=(+\mathrm{y},+\mathrm{x},+\mathrm{z}), Q_{3}=(+\mathrm{y},+\mathrm{z},+\mathrm{x})$, $\ldots, Q_{24}=(-\mathrm{x},+\mathrm{z},-\mathrm{y}), \ldots, Q_{48}=(-\mathrm{x},-\mathrm{z},-\mathrm{y})$. That is, for $Q_{24}$ as an instance, we have $\omega\left[q_{48}^{(1)}\right]=\mathrm{x}, \sigma\left[q_{48}^{(1)}\right]={ }^{-}-$', $\omega\left[q_{48}^{(2)}\right]=\mathrm{z}$, etc. Our representation ensures the following.

1. $\mathbb{C}_{a}=\left\{\mathbb{Q}_{b}: b=6(a-1)+c, c=1,2, \ldots, 6\right\}$.

2. Two q-octants $\mathbb{Q}_{i}$ and $\mathbb{Q}_{j}$ lie in the same c-octant if and only if $\lceil i / 6\rceil=\lceil j / 6\rceil$ (with $\mathbb{C}_{\lceil i / 6\rceil}$ as their common c-octant).

Equivalently, $\mathbb{Q}_{i}$ and $\mathbb{Q}_{j}$ lie in the same c-octant if and only if $\sigma\left[q_{i}\right]=\sigma\left[q_{j}\right]$ $\forall\left(q_{i}, q_{j}\right) \in\left\{\left(q_{i}^{\prime}, q_{j}^{\prime}\right):\left(\left(q_{i}^{\prime}, q_{j}^{\prime}\right) \in Q_{i} \times Q_{j}\right) \wedge\left(\omega\left[q_{i}^{\prime}\right]=\omega\left[q_{j}^{\prime}\right]\right)\right\}$. 
3. Let $w=0$ be one of the three coordinate planes, with $w \in\{x, y, z\}$. Then two c-octants $\mathbb{C}_{i}$ and $\mathbb{C}_{j}$ lie in two different half-spaces defined by $w=0$ if and only if the elements in $C_{i}$ and $C_{j}$ corresponding to $w$ are different.

Example 1. $C_{1}(+,+,+)$ and $C_{2}(-,+,+)$ have their 1st element different, which implies they are in two different half-spaces defined by the coordinate plane $x=0$; however, their 2 nd and 3rd elements being both ' + ', either of them lies in the half-space $y \geqslant 0$ and in the half-space $z \geqslant 0$.

\subsection{Metrics}

We define $x$-distance and $y$-distance between two (real or integer) points, $p(i, j$ ) and $p^{\prime}\left(i^{\prime}, j^{\prime}\right)$, as $d_{x}\left(p, p^{\prime}\right)=\left|i-i^{\prime}\right|$ and $d_{y}\left(p, p^{\prime}\right)=\left|j-j^{\prime}\right|$, respectively. In $\mathbb{R}^{3}$ or in $\mathbb{Z}^{3}$, we have also $z$-distance, given by $d_{z}\left(p, p^{\prime}\right)=\left|k-k^{\prime}\right|$, for $p(i, j, k)$ and $p^{\prime}\left(i^{\prime}, j^{\prime}, k^{\prime}\right)$. Using these inter-point distances, we define the respective $x-, y-$, and $z$-distances between a point $p(i, j, k)$ and a surface $S$ as follows. Let $d_{x}(p, S)$ be the $x$-distance between a point $p(i, j, k)$ and a surface $S$. If there exists a point $p^{\prime}\left(x^{\prime}, y^{\prime}, z^{\prime}\right)$ in $S$ such that $\left(y^{\prime}, z^{\prime}\right)=(j, k)$, then $d_{x}(p, S)=d_{x}\left(p, p^{\prime}\right)$; otherwise, $d_{x}(p, S)=\infty$. The other two distances, i.e., $d_{y}(p, S)$ and $d_{z}(p, S)$, are defined in a similar way; note that the metric $d_{z}(p, S)$ is not defined in 2D. These metrics are used to define the isothetic distance as follows.

Definition 1. Between two points $p_{1}\left(i_{1}, j_{1}\right)$ and $p_{2}\left(i_{2}, j_{2}\right)$, the isothetic distance is taken as the Minkowski norm [9], $d_{\infty}\left(p_{1}, p_{2}\right)=\max \left\{d_{x}\left(p_{1}, p_{2}\right), d_{y}\left(p_{1}, p_{2}\right)\right\}$; between a point $p(i, j)$ and a curve $C$, it is $d_{\perp}(p, C)=\min \left\{d_{x}(p, C), d_{y}(p, C)\right\}$, where $d_{x}(p, C)$ and $d_{y}(p, C)$ are defined similar to $d_{x}(p, S)$ and $d_{y}(p, S)$ respectively; between a $3 D$ point $p(i, j, k)$ and a surface $S$, it is $d_{\perp}(p, S)=\min \left\{d_{x}(p, S)\right.$, $\left.d_{y}(p, S), d_{z}(p, S)\right\}$.

\subsection{Topology}

A voxel is an integer point in 3D space, and equivalently, a 3-cell [9]. Two voxels are said to be 0 -adjacent if they share a vertex (0-cell), 1-adjacent if they share an edge (1-cell), and 2-adjacent if they share a face (2-cell). Thus, two distinct voxels, $p_{1}\left(i_{1}, j_{1}, k_{1}\right)$ and $p_{2}\left(i_{2}, j_{2}, k_{2}\right)$ are 1 -adjacent if and only if $\left|i_{1}-i_{2}\right|+$ $\left|j_{1}-j_{2}\right|+\left|k_{1}-k_{2}\right| \leqslant 2$ and $\max \left\{\left|i_{1}-i_{2}\right|,\left|j_{1}-j_{2}\right|,\left|k_{1}-k_{2}\right|\right\}=1$; 2-adjacent if and only if $\left|i_{1}-i_{2}\right|+\left|j_{1}-j_{2}\right|+\left|k_{1}-k_{2}\right|=1$; and 0 -adjacent if and only if $\left|i_{1}-i_{2}\right|=\left|j_{1}-j_{2}\right|=\left|k_{1}-k_{2}\right|=1$. Clearly, 0-adjacent (1-adjacent) voxels are not considered as adjacent while considering 1-neighborhood (2-neighborhood) connectivity. Note that the 0-, 1-, and 2-neighborhood notations, as adopted in this paper and also in [15], correspond respectively to the classical 26-, 18-, and 6-neighborhood notations used in [6].

Based on above definitions, a digital sphere is said to be 2-separating if it does not contain any 2-tunnel, that is, its interior and exterior are not connected by a 2-connected path [6]. A 2-separating digital sphere is irreducible if and only if it does not contain any simple voxel, that is, removal of any voxel violates its 
topological property of 2-separableness [6]. We use $S_{r}^{\mathbb{R}}$ to denote the real sphere of radius $r$ and centered at $o$, use $S_{r}^{\mathbb{Z}_{1}}$ to denote the part of $S_{r}^{\mathbb{Z}}$ lying in $\mathbb{Q}_{1}$, and use $p \in S_{r}^{\mathbb{Z}}$ when a voxel $p$ belongs to (voxel set) $S_{r}^{\mathbb{Z}}$. Our work is based on the following definition of digital sphere.

Definition 2. A digital sphere $S_{r}^{\mathbb{Z}}$ is an irreducible 2-separating subset of the voxel set having isothetic distance less than $\frac{1}{2}$ from $S_{r}^{\mathbb{R}}$.

Note that in [15, the only strict k-separating or irreducible digital sphere results from outer Gaussian digitization, but its voxels are not limited by a maximum isothetic distance of $\frac{1}{2}$ from $S_{r}^{\mathbb{R}}$. The closed centered 2-separating digitized sphere is another proposition in [15], which is not necessarily irreducible.

\subsection{Characterization}

The characterization of $S_{r}^{\mathbb{Z}}$ is required to decide in constant time whether a particular voxel $(i, j, k)$ belongs to $S_{r}^{\mathbb{Z}}$. We start with the following lemmas.

Lemma 1. $d_{\perp}\left(p, S_{r}^{\mathbb{R}}\right)=\left|k-\sqrt{r^{2}-\left(i^{2}+j^{2}\right)}\right| \forall p(i, j, k) \in S_{r}^{\mathbb{Z}_{1}}$.

Proof. Let $p(i, j, k) \in S_{r}^{\mathbb{Z}_{1}}$, and $(x, j, k),(i, y, k)$, and $(i, j, z)$ be the respective points on $S_{r}^{\mathbb{R}}$ taken along the lines parallel to $x$-, $y$-, and $z$-axes, and passing through $p$. Observe that the points $(x, j, k)$ and $(i, y, k)$ may be nonexistent, but the point $(i, j, z)$ always exists. If all three exist, then

$x^{2}+j^{2}+k^{2}=i^{2}+y^{2}+k^{2}=i^{2}+j^{2}+z^{2}=r^{2}$, or, $k^{2}-z^{2}=j^{2}-y^{2}=i^{2}-x^{2}$

or, $(k+z)(k-z)=(j+y)(j-y)=(i+x)(i-x)$.

In $\mathbb{Q}_{1}, i \leqslant j \leqslant k$ and $x \leqslant y \leqslant z$, or, $i+x \leqslant j+y \leqslant k+z$; so, from Eq. 1 ,

$$
|k-z| \leqslant|j-y| \leqslant|i-x| \text {. }
$$

If one or both $(x, j, k)$ and $(i, y, k)$ do not exist, then also $|k-z|$ remains the minimum. Hence, from Eq. 2, $d_{\perp}\left(p, S_{r}^{\mathbb{R}}\right)=|k-z|=\left|k-\sqrt{r^{2}-\left(i^{2}+j^{2}\right)}\right|$.

Lemma 2. $d_{\perp}\left(p, S_{r}^{\mathbb{R}}\right)<\frac{1}{2} \forall p \in S_{r}^{\mathbb{Z}}$.

Proof. If possible, let, w.l.o.g., $p(i, j, k) \in S_{r}^{\mathbb{Z}_{1}}$, such that $\left|k-\sqrt{r^{2}-\left(i^{2}+j^{2}\right)}\right|=$ $\frac{1}{2}$, or, w.l.o.g., $k-\sqrt{r^{2}-\left(i^{2}+j^{2}\right)}=-\frac{1}{2}$, which implies $S_{r}^{\mathbb{R}}$ has $p^{\prime}\left(i, j, k+\frac{1}{2}\right)$ as the point of intersection in $\mathbb{Q}_{1}$ with the $3 \mathrm{D}$ straight line $(x=i, y=j)$. Since $\left(i, j, k+\frac{1}{2}\right)$ lies on $S_{r}^{\mathbb{R}}$, we have $i^{2}+j^{2}+\left(k+\frac{1}{2}\right)^{2}=r^{2}$, which is a contradiction, since $r, i, j, k$ are all integers.

Lemma 2 helps in characterizing a voxel $p \in S_{r}^{\mathbb{Z}}$, as stated next.

Theorem 1. $p(i, j, k) \in S_{r}^{\mathbb{Z}}$ if and only if $p$ is not simple and $i^{2}+j^{2}+k^{2} \in$ $\left[r^{2}-\max \{|i|,|j|,|k|\}, r^{2}+\max \{|i|,|j|,|k|\}-1\right]$. 
Proof. Let, w.l.o.g., $p \in \mathbb{Q}_{1}$. So, $\max \{|i|,|j|,|k|\}=k$. Hence, by Lemma 1 and Lemma 2, $p \in S_{r}^{\mathbb{Z}_{1}}$ if and only if $p$ is not simple and

$$
\begin{aligned}
& -\frac{1}{2}<k-\sqrt{r^{2}-\left(i^{2}+j^{2}\right)}<\frac{1}{2} \\
\Leftrightarrow & k^{2}-k+\frac{1}{4}<r^{2}-\left(i^{2}+j^{2}\right)<k^{2}+k+\frac{1}{4} .
\end{aligned}
$$

Since $k^{2}-k, r^{2}-\left(i^{2}+j^{2}\right), k^{2}+k$ are integers, Eq. 4 is true if and only if

$$
\begin{aligned}
& k^{2}-k<r^{2}-\left(i^{2}+j^{2}\right) \leqslant k^{2}+k \\
\Leftrightarrow & r^{2}-k \leqslant i^{2}+j^{2}+k^{2}<r^{2}+k,
\end{aligned}
$$

and hence the proof for 1st q-octant. For other q-octants, the proof is similar.

Now, to obtain the necessary and sufficient condition of deciding whether a voxel is simple, we need the following theorem.

Theorem 2. A voxel $p(i, j, k)$ with $d_{\perp}\left(p, S_{r}^{\mathbb{R}}\right)<\frac{1}{2}$ is simple if and only if $i^{2}+$ $j^{2}+k^{2}=r^{2}+\max \{|i|,|j|,|k|\}-1$ and $\operatorname{mid}\{|i|,|j|,|k|\}=\max \{|i|,|j|,|k|\}$, where $\operatorname{mid}\{\cdot\}$ denotes the median element.

Proof. As in the proof of Theorem[1, let, w.l.o.g., $p \in \mathbb{Q}_{1} ; \operatorname{so}, \operatorname{mid}\{|i|,|j|,|k|\}=j$ and $\max \{|i|,|j|,|k|\}=k$. Let also, $d_{\perp}\left(p, S_{r}^{\mathbb{R}_{1}}\right)<\frac{1}{2}$, which implies $p$ satisfies Eq. 5 by Lemma 1 and Lemma 2 .

Now, we prove that $p(i, j, k)$ lies on $S_{r}^{\mathbb{Z}_{1}}$ and cannot be a simple voxel if $j<k$. For this, first observe that $(i, j, k-1)$ and $(i, j, k+1)$ lie in $\mathbb{Q}_{1}$, as $j \leqslant k-1$. Next, observe that for any $\left(i^{\prime}, j^{\prime}\right) \in \mathbb{Z}^{2}$, there can be at most one integer value of $k^{\prime}$ so that $\left(i^{\prime}, j^{\prime}, k^{\prime}\right)$ satisfies Eq. 3. This implies that $(i, j, k-1)$ lies in the interior and $(i, j, k+1)$ in the exterior of $S_{r}^{\mathbb{Z}}$. Hence, discarding $p$ would violate the 2-separableness of $S_{r}^{\mathbb{Z}}$.

Now, the conditions $i^{2}+j^{2}+k^{2}=r^{2}+\max \{|i|,|j|,|k|\}-1$ and $\operatorname{mid}\{|i|,|j|,|k|\}=$ $\max \{|i|,|j|,|k|\}$ imply $\left(i^{2}+k^{2}+k^{2}\right)=\left(r^{2}+k-1\right)$, which is true if and only if

$$
\begin{aligned}
& \left(i^{2}+(k-1)^{2}+k^{2}\right)=\left(r^{2}-k\right) \\
\Leftrightarrow & (i, k-1, k) \in S_{r}^{\mathbb{Z}} \text { by Theorem 1] and }(i, k, k-1) \in S_{r}^{\mathbb{Z}} \\
\Leftrightarrow & (i, k, k) \text { is simple. }
\end{aligned}
$$

For $p$ lying in some other octant, the proof follows a similar way.

Using Theorem 1 and Theorem 2], we get a mathematically refined definition of digital sphere, as stated in the following theorem.

Theorem 3. The voxel set of the digital sphere $S_{r}^{\mathbb{Z}}$ is given by

$$
\left\{\begin{array}{c}
(i, j, k) \in \mathbb{Z}^{3}: r^{2}-\max \{|i|,|j|,|k|\} \leqslant i^{2}+j^{2}+k^{2}<r^{2}+\max \{|i|,|j|,|k|\} \\
\wedge\left(\begin{array}{c}
\left(i^{2}+j^{2}+k^{2} \neq r^{2}+\max \{|i|,|j|,|k|\}-1\right) \\
\vee(\operatorname{mid}\{|i|,|j|,|k|\} \neq \max \{|i|,|j|,|k|\})
\end{array}\right)
\end{array}\right\} .
$$




\section{Discrete Spherical Geodesic Path and Circle}

Theorem 3 is used to decide in constant time whether a voxel $p(i, j, k)$ belongs to $S_{r}^{\mathbb{Z}}$. For generating the discrete spherical geodesic path $\boldsymbol{\pi}_{r}^{\mathbb{Z}}(s, t)$ from a voxel $s \in S_{r}^{\mathbb{Z}}$ to a voxel $t \in S_{r}^{\mathbb{Z}}$, we consider the real plane $\Pi_{r}^{\mathbb{R}}(s, t)$ that passes through $s, t$, and the center of $S_{r}^{\mathbb{Z}}$. Considering voxels as 3-cells, let $I_{r}^{\mathbb{Z}}(s, t)$ be the set of voxels of $S_{r}^{\mathbb{Z}}$ intersected by $\Pi_{r}^{\mathbb{R}}(s, t)$. We have the following lemma for $I_{r}^{\mathbb{Z}}(s, t)$.

Lemma 3. $d_{\perp}\left(p, \Pi_{r}^{\mathbb{R}}(s, t)\right) \leqslant \frac{3}{2} \forall p \in I_{r}^{\mathbb{Z}}(s, t)$.

Proof. Let $\delta_{e}:=d_{e}\left(p, \Pi_{r}^{\mathbb{R}}(s, t)\right)$ be the real (Euclidean) distance of the point $p$ from $\Pi_{r}^{\mathbb{R}}(s, t)$. If $\Pi_{r}^{\mathbb{R}}(s, t)$ intersects the voxel $p$, then $\delta_{e} \leqslant \frac{\sqrt{3}}{2}$.

Now, let $\delta_{x}=d_{x}\left(p, \Pi_{r}^{\mathbb{R}}(s, t)\right), \delta_{y}=d_{y}\left(p, \Pi_{r}^{\mathbb{R}}(s, t)\right), \delta_{z}=d_{z}\left(p, \Pi_{r}^{\mathbb{R}}(s, t)\right)$. Observe that $\delta_{x}=\frac{\delta_{e}}{\cos \theta_{x}}, \delta_{y}=\frac{\delta_{e}}{\cos \theta_{y}}, \delta_{z}=\frac{\delta_{e}}{\cos \theta_{z}}$, where, $\cos ^{2} \theta_{x}+\cos ^{2} \theta_{y}+\cos ^{2} \theta_{z}=1$. Here, $\cos \theta_{x}$ is the angle between the $x$-axis-parallel line through $p$ and the perpendicular on $\Pi_{r}^{\mathbb{R}}(s, t)$ dropped from $p$, etc. So, the supremum of $d_{\perp}\left(p, \Pi_{r}^{\mathbb{R}}(s, t)\right):=$ $\min \left\{\delta_{x}, \delta_{y}, \delta_{z}\right\}$ corresponds to the infimum of the largest element in $C_{\theta}:=\left\{\cos \theta_{x}, \cos \theta_{y}, \cos \theta_{z}\right\}$, and hence to the infimum of the largest element in $C_{\theta}^{(2)}:=\left\{\cos ^{2} \theta_{x}, \cos ^{2} \theta_{y}, \cos ^{2} \theta_{z}\right\}$, subject to $\cos ^{2} \theta_{x}+\cos ^{2} \theta_{y}+\cos ^{2} \theta_{z}=1$. Clearly, the largest element in $C_{\theta}^{(2)}$ is at least $\frac{1}{3}$, or, the largest element in $C_{\theta}$ is at least $\frac{1}{\sqrt{3}}$, whence $d_{\perp}\left(p, \Pi_{r}^{\mathbb{R}}(s, t)\right) \leqslant \delta_{e} / \frac{1}{\sqrt{3}}=\frac{3}{2}$.

Theorem 4. For any two voxels $s \in S_{r}^{\mathbb{Z}}$ and $t \in S_{r}^{\mathbb{Z}}$, there always exist two 1connected paths, $\pi_{r}^{\mathbb{Z}}(s, t)^{\prime} \subset I_{r}^{\mathbb{Z}}(s, t)$ and $\pi_{r}^{\mathbb{Z}}(t, s)^{\prime \prime} \subset I_{r}^{\mathbb{Z}}(s, t)$, such that $\pi_{r}^{\mathbb{Z}}(s, t)^{\prime} \cup$ $\pi_{r}^{\mathbb{Z}}(t, s)^{\prime \prime}$ forms a 1-connected simple cycle in $I_{r}^{\mathbb{Z}}(s, t)$.

Proof. Given a continuous surface $A$, there is a unique supercover of $A$, defined as the set of all voxels intersecting $A\left[6\right.$. Hence, if $\Pi_{r}^{\mathbb{Z}}(s, t)$ denotes the supercover of $\Pi_{r}^{\mathbb{R}}(s, t)$, then all the voxels - conceived as 3-cells - that are intersected by $\Pi_{r}^{\mathbb{R}}(s, t)$, comprise the set $\Pi_{r}^{\mathbb{Z}}(s, t)$. As shown in [2], the supercover of a plane is 2 -separable.

We define $\mathcal{S}_{r^{-}}^{\mathbb{Z}}$ and $\mathcal{S}_{r^{+}}^{\mathbb{Z}}$ as the respective interior and exterior of $S_{r}^{\mathbb{Z}}$. So, by Definition 2, the sets $\mathcal{S}_{r^{\mathbb{Z}}}^{\mathbb{Z}}$ and $\mathcal{S}_{r^{+}}^{\mathbb{Z}}$ are disconnected in 2-neighborhood. Also, let $\Pi_{r^{-}}^{\mathbb{Z}}(s, t)=\Pi_{r}^{\mathbb{Z}}(s, t) \cap \mathcal{S}_{r^{-}}^{\mathbb{Z}}$ and $\Pi_{r^{+}}^{\mathbb{Z}}(s, t)=\Pi_{r}^{\mathbb{Z}}(s, t) \cap \mathcal{S}_{r^{+}}^{\mathbb{Z}}$. Note that $\Pi_{r^{-}}^{\mathbb{Z}}(s, t)$ is a non-empty set and always contains $o$ for $r \geqslant 1$, since $\Pi_{r}^{\mathbb{R}}(s, t)$ passes through $o$. This yields

$$
\Pi_{r}^{\mathbb{Z}}(s, t)=\Pi_{r^{-}}^{\mathbb{Z}}(s, t) \cup I_{r}^{\mathbb{Z}}(s, t) \cup \Pi_{r^{+}}^{\mathbb{Z}}(s, t)
$$

where, $\Pi_{r^{-}}^{\mathbb{Z}}(s, t), I_{r}^{\mathbb{Z}}(s, t)$, and $\Pi_{r^{+}}^{\mathbb{Z}}(s, t)$ are pairwise disjoint.

Now, as $\mathcal{S}_{r^{-}}^{\mathbb{Z}}$ and $\mathcal{S}_{r^{+}}^{\mathbb{Z}}$ are not 2-connected, their respective subsets $\Pi_{r^{-}}^{\mathbb{Z}}(s, t)$ and $\Pi_{r^{+}}^{\mathbb{Z}}(s, t)$ are also not 2-connected. So, by Eq. [6, the set $I_{r}^{\mathbb{Z}}(s, t)$ forms a 2-separating set between $\Pi_{r^{-}}^{\mathbb{Z}}(s, t)$ and $\Pi_{r^{+}}^{\mathbb{Z}}(s, t)$, or, equivalently, $I_{r}^{\mathbb{Z}}(s, t)$ is a 1 -connected set that also 2 -separates $S_{r}^{\mathbb{Z}}$. Therefore, there always exists a 1connected simple path $\pi_{r}^{\mathbb{Z}}(s, t)^{\prime} \in I_{r}^{\mathbb{Z}}(s, t)$ from $s$ to $t$, and another 1-connected simple path $\pi_{r}^{\mathbb{Z}}(t, s)^{\prime \prime} \in I_{r}^{\mathbb{Z}}(s, t)$ from $t$ to $s$, where $\pi_{r}^{\mathbb{Z}}(s, t)^{\prime} \cap \pi_{r}^{\mathbb{Z}}(t, s)^{\prime \prime}=\{s, t\}$. Hence, there always exists a 1 -connected simple cycle $\left(\pi_{r}^{\mathbb{Z}}(s, t)^{\prime} \cup \pi_{r}^{\mathbb{Z}}(t, s)^{\prime \prime}\right)$ in $I_{r}^{\mathbb{Z}}(s, t)$ containing any two voxels $s \in S_{r}^{\mathbb{Z}}$ and $t \in S_{r}^{\mathbb{Z}}$. 
From Theorem 4, it is clear that for two given voxels $s \in S_{r}^{\mathbb{Z}}$ and $t \in S_{r}^{\mathbb{Z}}$, we get at least two 1 -connected paths, $\pi_{r}^{\mathbb{Z}}(s, t)^{\prime}$ and $\pi_{r}^{\mathbb{Z}}(s, t)^{\prime \prime}$, in $I_{r}^{\mathbb{Z}}(s, t)$, having no voxels in common, excepting $s$ and $t$. The discrete 3D (integer) circle passing through two given voxels $s$ and $t$ is, therefore, given by $C_{r}^{\mathbb{Z}}(s, t)=\pi_{r}^{\mathbb{Z}}(s, t)^{\prime} \cup$ $\pi_{r}^{\mathbb{Z}}(t, s)^{\prime \prime}$. Note that specifying only $s \in S_{r}^{\mathbb{Z}}$ and $t \in S_{r}^{\mathbb{Z}}$ would suffice to get $\boldsymbol{\pi}_{r}^{\mathbb{Z}}(s, t)$, and hence $C_{r}^{\mathbb{Z}}(s, t)$, since a unique value of $r$ would satisfy Theorem 1 for each of $s$ and $t$.

\section{Algorithm DSGP}

We define inter-octant distance $d_{i, j}^{(8)}$ corresponding to $\mathbb{C}_{i}$ and $\mathbb{C}_{j}$. With $s \in \mathbb{C}_{i}$ and $t \in \mathbb{C}_{j}$, it is given by the count of q-octants crossed by $\boldsymbol{\pi}_{r}^{\mathbb{Z}}(s, t)$ before entering $\mathbb{C}_{j}$. Mathematically,

$$
d_{i, j}^{(8)}=1+\sum_{u=1}^{3} 2^{u-1}\left(c_{i}^{(u)} \oplus c_{j}^{(u)}\right)
$$

where, $c_{i}^{(u)} \oplus c_{j}^{(u)}=1$ if $c_{i}^{(u)} \neq c_{j}^{(u)}$, and 0 otherwise. If $i=j$, then $d_{i, j}^{(8)}=0$; otherwise, the value of $d_{i, j}^{(8)}$ lies in the interval $[1,7]$. The maximum value $d_{i, j}^{(8)}=$ 7 is obtained when $\mathbb{C}_{i}$ and $\mathbb{C}_{j}$ are diametrically opposite, i.e., $c_{i}^{(u)} \neq c_{j}^{(u)}$ for $u=1,2,3$. The pair $(s, t)$ becomes antipodal if their c-octants are diametrically opposite and $s, o, t$ are collinear. Then $\Pi_{r}^{\mathbb{R}}(s, t)$ has no fixed orientation, and so a third point $q$ on $S_{r}^{\mathbb{Z}}$ needs to be specified, which would lie in $\boldsymbol{\pi}_{r}^{\mathbb{Z}}(s, t)$.

Similarly, we define the intra-octant distance $d_{i, j}^{(6)}$ between two q-octants, $\mathbb{Q}_{i}$ and $\mathbb{Q}_{j}$, when they lie in same c-octant. It provides the count of q-octants containing the geodesic path from any point $s \in \mathbb{Q}_{i}$ to any point $t \in \mathbb{Q}_{j}$. According to our representation, it is given by one plus the minimum number of swaps among the elements in $Q_{i}$, so that, after swaps, the transformed 3-tuple is identical with $Q_{j}$. Two elements are swapped in $Q_{i}$ or in any of its intermediate configurations only if the elements are consecutive in $Q_{i}$ or in that configuration (i.e., 3-tuple). Using $d_{i, j}^{(8)}$ and $d_{i, j}^{(6)}$, we compute the $q$-octant distance $d_{i, j}^{(48)}$ between $s$ and $t$. It gives the count of q-octants containing the geodesic path from $s$ to $t$, irrespective of their positions on the sphere. Its measure turns out to be

$$
d_{i, j}^{(48)}=d_{i, j}^{(8)}+d_{i, j}^{(6)}-1
$$

Combining the above, we simplify the rule of determining the sequence of qoctants containing $\boldsymbol{\pi}_{r}^{\mathbb{Z}}(s, t)$ as follows. Let $\mathbb{Q}_{i}$ and $\mathbb{Q}_{j}$ be the q-octants containing $s$ and $t$, respectively. Then the sequence of q-octants through which $\boldsymbol{\pi}_{r}^{\mathbb{Z}}(s, t)$ passes, is given by a/the minimum-length sequence of transformations applied on $Q_{i}$ to attain the configuration $Q_{j}$. Following are the rules of transformation.

$T_{1}$. Change the sign of the first element $q_{i}^{(1)}$ in $Q_{i}$ (or its intermediate configuration) only if $\sigma\left[q_{i}^{(1)}\right] \neq \sigma\left[q_{j}^{(1)}\right]$. This signifies transition from one half-space (or, c-octant) to another half-space. 
$T_{2}$. Swap two elements in $Q_{i}$ (or its intermediate configuration) only if they are consecutive. This signifies transition from one q-octant to its adjacent q-octant.

From the sequence of q-octants obtained by the required transformations, we determine the q-octant $\mathbb{Q}_{i^{\prime}}$ immediately next to the q-octant $\mathbb{Q}_{i}$ of $s$. We use the 3-tuples corresponding to $\mathbb{Q}_{i}$ and $\mathbb{Q}_{i^{\prime}}$ for computing the direction vector $\mathbf{d}_{s}:=\left(d_{s}^{(1)}, d_{s}^{(2)}, d_{s}^{(3)}\right) \in\{+1,-1, \pm 1\}^{3}$. It is required to find the candidate voxels that are 1-adjacent to $s\left(\mathbb{A}_{1}(s)\right)$, belong to $I_{r}^{\mathbb{Z}}(s, t)$, and is directed towards the shorter between the two possible geodesics from $s$ to $t$ (Theorem 4). The elements $d_{s}^{(1)}, d_{s}^{(2)}, d_{s}^{(3)}$ correspond to the moves along $x-, y$-, $z$-axes, respectively. The notation +1 signifies that there can be a unit move or no move (from $s$ ) along the positive axis of the corresponding coordinate; similarly, -1 signifies a unit move or no move along the negative axis, and \pm 1 signifies no move or a unit move along positive or negative axis. In case of more than one minimum-length sequence of q-octants from $\mathbb{Q}_{i}$ to $\mathbb{Q}_{j}$, we consider the q-octant nearest to $\mathbb{Q}_{i}$ and common to these sequences, for computing $\mathbf{d}_{s}$. The rationale is that only one of these sequences would be intersected by $\Pi_{r}^{\mathbb{R}}(s, t)$, and hence the q-octant common to these sequences is used. The following examples clarify the idea.

Example 2. See Fig. 2. Given $s(10,-2,6) \in \mathbb{Q}_{15}$ and $t(-3,10,6) \in \mathbb{Q}_{12}$, their respective 3-tuples are $Q_{15}:=(-\mathrm{y},+\mathrm{z},+\mathrm{x})$ and $Q_{12}:=(-\mathrm{x},+\mathrm{z},+\mathrm{y})$. The minimum-length sequence of transformations corresponding to $\boldsymbol{\pi}_{r}^{\mathbb{Z}}(s, t)$ is:

$(-\mathrm{y},+\mathrm{z},+\mathrm{x}) \stackrel{T_{1}}{\longrightarrow}(+\mathrm{y},+\mathrm{z},+\mathrm{x}) \stackrel{T_{2}}{\longrightarrow}(+\mathrm{y},+\mathrm{x},+\mathrm{z}) \stackrel{T_{2}}{\longrightarrow}(+\mathrm{x},+\mathrm{y},+\mathrm{z}) \stackrel{T_{2}}{\longrightarrow}(+\mathrm{x},+\mathrm{z},+\mathrm{y})$ $\stackrel{T_{1}}{\longrightarrow}(-\mathrm{x},+\mathbf{z},+\mathrm{y})$, or, $Q_{15} \stackrel{T_{1}}{\longrightarrow} Q_{3} \stackrel{T_{2}}{\longrightarrow} Q_{2} \stackrel{T_{2}}{\longrightarrow} Q_{1} \stackrel{T_{2}}{\longrightarrow} Q_{6} \stackrel{T_{1}}{\longrightarrow} Q_{12}$.

Notice that there is another minimum-length sequence: $(-\mathrm{y},+\mathrm{z},+\mathrm{x}) \stackrel{T_{1}}{\longrightarrow}(+\mathrm{y},+\mathrm{z},+\mathrm{x})$ $\stackrel{T_{2}}{\longrightarrow}(+\mathrm{z},+\mathrm{y},+\mathrm{x}) \stackrel{T_{2}}{\longrightarrow}(+\mathrm{z},+\mathrm{x},+\mathrm{y}) \stackrel{T_{2}}{\longrightarrow}(+\mathrm{x},+\mathrm{z},+\mathrm{y}) \stackrel{T_{2}}{\longrightarrow}(-\mathrm{x},+\mathrm{z},+\mathrm{y})$, which implies $Q_{15} \stackrel{T_{1}}{\longrightarrow} Q_{3} \stackrel{T_{2}}{\longrightarrow} Q_{4} \stackrel{T_{2}}{\longrightarrow} Q_{5} \stackrel{T_{2}}{\longrightarrow} Q_{6} \stackrel{T_{2}}{\longrightarrow} Q_{12}$.

Either of these implies that the q-octant next to $\mathbb{Q}_{15}$ through which $\boldsymbol{\pi}_{r}^{\mathbb{Z}}(s, t)$ passes, is $\mathbb{Q}_{3}$. Since $Q_{15}=(-\mathrm{y},+\mathrm{z},+\mathrm{x})$ and $Q_{3}=(+\mathrm{y},+\mathrm{z},+\mathrm{x})$, the $y$-coordinate of each voxel $p \in \mathbb{A}_{1}(s) \cap I_{r}^{\mathbb{Z}}(s, t)$, cannot ever decrease. On the contrary, the $x$ - and the $z$-coordinates of $p$ have no such restriction. Hence, the direction vector $\mathbf{d}_{s}$ is chosen as $( \pm 1,+1, \pm 1)$.

Example 3. Let $s \in \mathbb{Q}_{1}$ and $t \in \mathbb{Q}_{4}$. So, $Q_{1}=(+\mathrm{x},+\mathrm{y},+\mathrm{z})$ and $Q_{4}=(+\mathbf{z},+\mathrm{y},+\mathrm{x})$. We have two minimum-length sequence of transformations: (i) $Q_{1} \stackrel{T_{2}}{\longrightarrow} Q_{2} \stackrel{T_{2}}{\longrightarrow} Q_{3} \stackrel{T_{2}}{\longrightarrow} Q_{4}$; (ii) $Q_{1} \stackrel{T_{2}}{\longrightarrow} Q_{6} \stackrel{T_{2}}{\longrightarrow} Q_{5} \stackrel{T_{2}}{\longrightarrow} Q_{4}$.

Contrary to Example 2, here the q-octants following $\mathbb{Q}_{1}$ in two cases are different: $\mathbb{Q}_{2}$ for (i) and $\mathbb{Q}_{6}$ for (ii). So, we look ahead until there is a matching q-octant, i.e., $\mathbb{Q}_{4}$ in this case. We compute $\mathbf{d}_{s}$ as the relative shifts in positions of the coordinate values in $Q_{4}$. In $Q_{1}$, the 1 st element is $+\mathrm{x}$, which is shifted to 3 rd position in $Q_{4}$. So, the 1 st element in $\mathbf{d}_{s}$ becomes +1 , and by similar reasoning with the 2 nd and the 3 rd elements, we get $\mathbf{d}_{s}=(+1, \pm 1,-1)$.

Analysis. See Algorithm 1 and its demonstration in Fig. 2, The adjacency list $L$ of the underlying undirected graph $G(V, E)$ is prepared based on 1-adjacency of the voxels in $I_{r}^{\mathbb{Z}}(s, t)$. The vertices adjacent to each $u \in V$ are inserted in the 

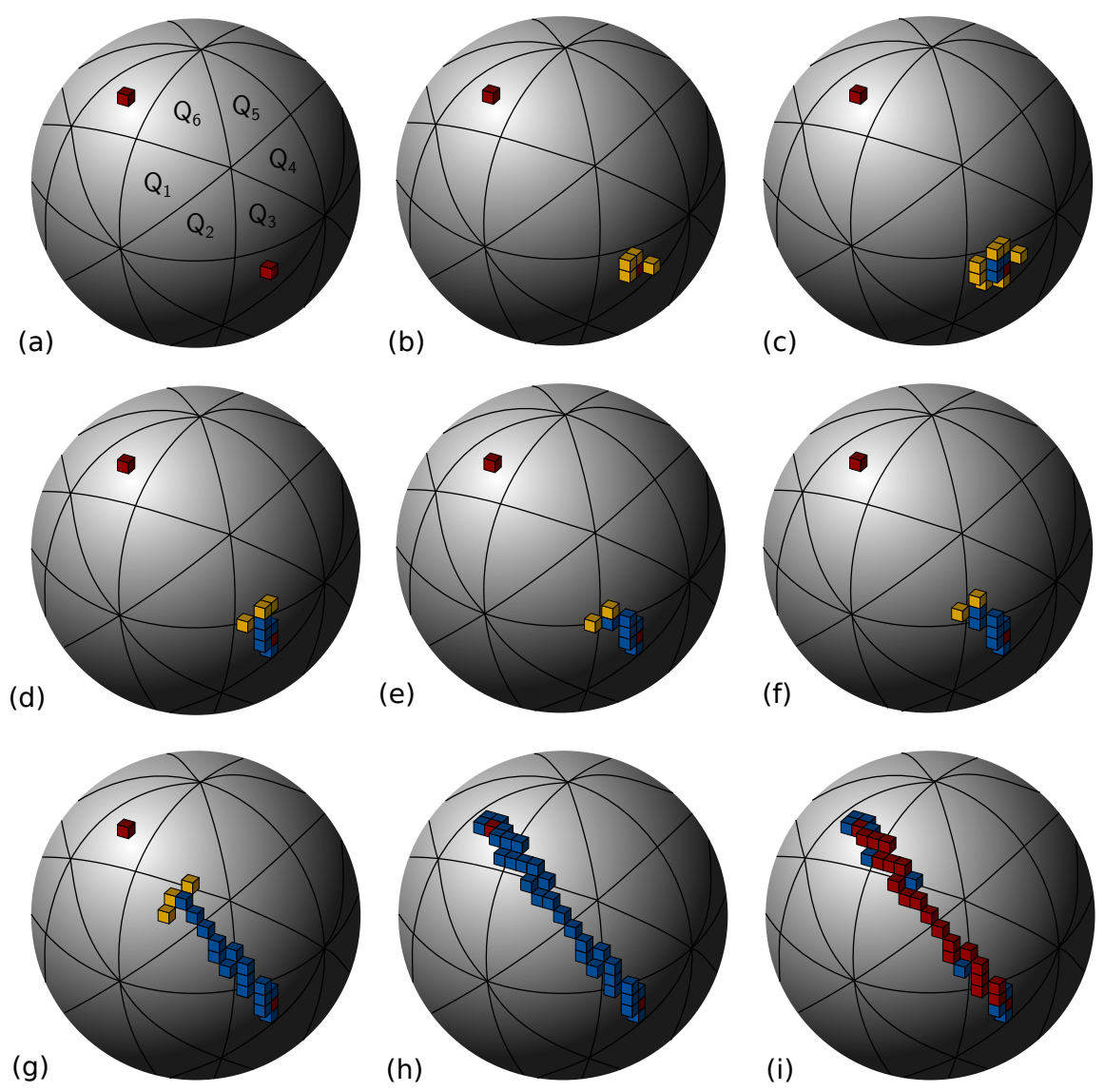

Fig. 2. A demonstration of the proposed algorithm for $r=12$. (a) $s(10,-2,6) \in \mathbb{Q}_{15}$, $t(-3,10,6) \in \mathbb{Q}_{12}$. (b) Yellow: $S_{r}^{\mathbb{Z}} \cap \mathbb{A}_{1}(s)$. (c) Blue: $S_{r}^{\mathbb{Z}} \cap \mathbb{A}_{1}(s) \cap I_{r}^{\mathbb{Z}}(s, t)$, Yellow: $\{p \in$ $\mathbb{A}_{1}(q): q$ is Blue\}. (d-h) Blue: Progress of Procedure MakeAdjList for $I_{r}^{\mathbb{Z}}(s, t)$. (i) Red: $\boldsymbol{\pi}_{r}^{\mathbb{Z}}(s, t) \subset I_{r}^{\mathbb{Z}}(s, t)$.

adjacency chain $L[u]$ of $u$ in non-increasing order of their isothetic distances from $\Pi_{r}^{\mathbb{R}}(s, t)$, (MakeAdjList, Line 9). This is needed to maintain locally minimum isothetic distance from $\Pi_{r}^{\mathbb{R}}(s, t)$ while running Prioritized-BFS (Algorithm 1 , Line 3). In Line 8 of MakeAdjList, Theorem 1 is used to determine the voxels that are 1-adjacent with the current voxel and belong to $S_{r}^{\mathbb{Z}}$, in constant time. Thus, MakeAdjList and Prioritized-BFS consumes $O(n)$ time each, where $n$ is the number of voxels comprising $\boldsymbol{\pi}_{r}^{\mathbb{Z}}(s, t)$. The direction vector $\mathbf{d}_{s}$ is computed from the sequence(s) in no more than $O(n)$ time complexity. Hence, the total time complexity of Algorithm DSGP is linear in the length of $\boldsymbol{\pi}_{r}^{\mathbb{Z}}(s, t)$.

\section{Results}

The proposed algorithm is implemented in $\mathrm{C}$ in Ubuntu 12.04 32-bit, Kernel Linux 3.2.0-31-generic-pae, GNOME 3.4.2, Intel ${ }^{\circledR}$ Core ${ }^{\mathrm{TM}}$ i5-2400 CPU 3.10GHz. 

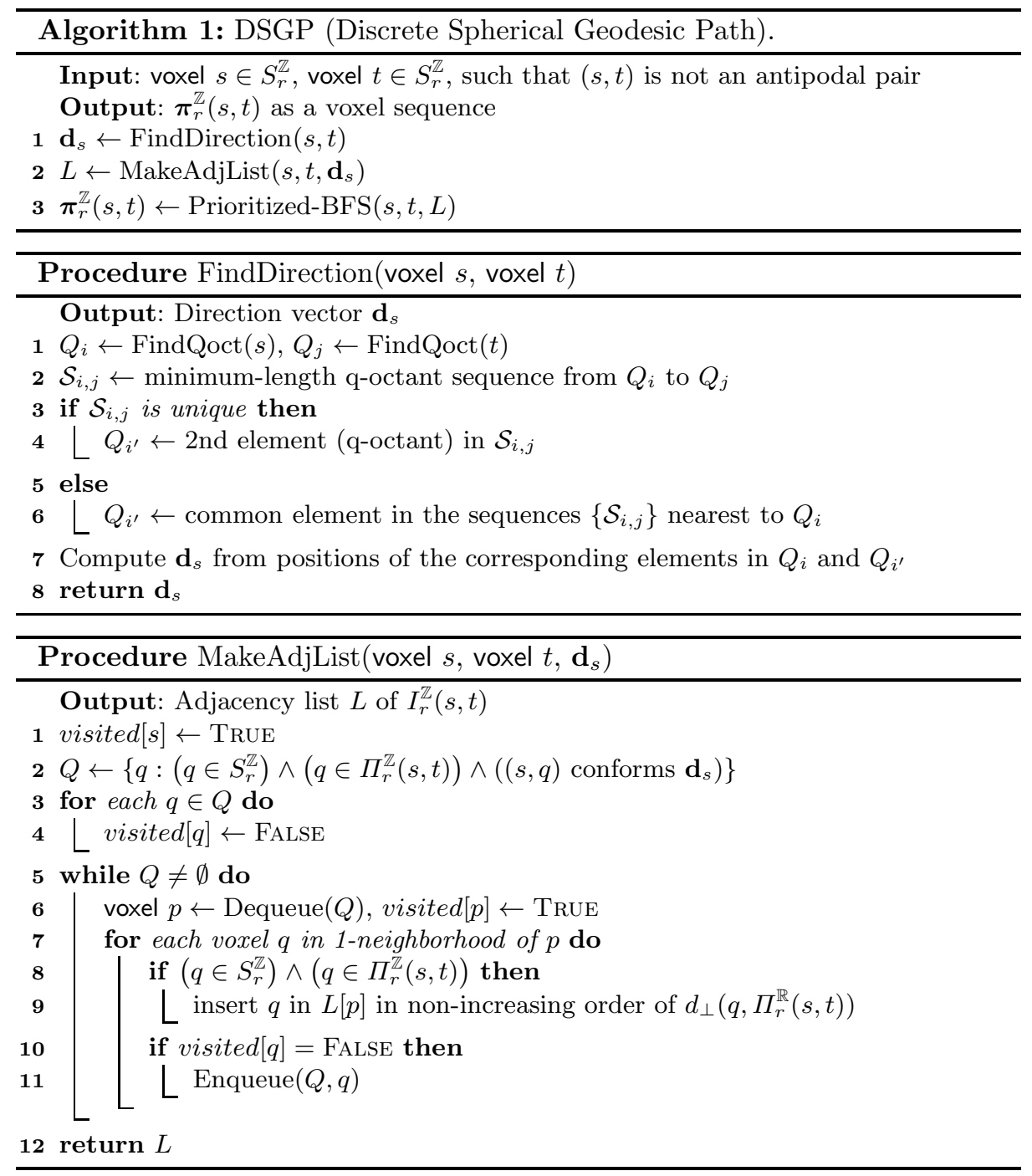

As the algorithm is of linear time complexity and readily implementable with primitive operations in the integer space, it computes the spherical geodesic paths and $3 \mathrm{D}$ circles in $\mathbb{Z}^{3}$ quite fast and efficiently. To demonstrate this, a summary of some experimental results is given in Appendix. For radius $r$ ranging from 10 to 1000, different source and destination points are chosen, and their geodesic paths are computed. For each path $\boldsymbol{\pi}_{r}^{\mathbb{Z}}(s, t)$, its length $\left|\boldsymbol{\pi}_{r}^{\mathbb{Z}}(s, t)\right|$, measured in terms of number of voxels comprising the path, is shown, along with the corresponding q-octant distance, $d_{i, j}^{(48)}$. The CPU time, measured in milliseconds, reflects the linear-time behavior of the algorithm, as explained in Section 4

The figure in Appendix shows a set of discrete spherical geodesics and their corresponding circles produced by the algorithm. Note that a discrete geodesic 
circle can be obtained by taking the union of the path $\boldsymbol{\pi}_{r}^{\mathbb{Z}}(s, t)$ with its complementary path, i.e., $\boldsymbol{\pi}_{r}^{\mathbb{Z}}(t, s)$, taken in the same order of cyclic movement. Clearly, such a circle would always include $s$ and $t$. However, the inclusion of $t$ is not ensured if we ignore $t$ during Prioritized-BFS and moves forward until the traversal returns to $s$, although the resultant geodesic circle would comprise voxels lying within an isothetic distance of $\frac{3}{2}$ from $\Pi_{r}^{\mathbb{R}}(s, t)$.

\section{Conclusion}

We have shown how number-theoretic characterization helps in developing efficient algorithms related to discrete geodesics on a spherical surface. The problems of finding iso-contours and of geodesic distance query, defined and attempted in 3D real space [17, 18, are also pertinent in 3D digital space. The technique introduced in this paper may be extended to solve such problems with efficiency and theoretical guarantee.

\section{References}

[1] Balasubramanian, M., Polimeni, J.R., Schwartz, E.L.: Exact geodesics and shortest paths on polyhedral surfaces. IEEE TPAMI 31, 1006-1016 (2009)

[2] Brimkov, V., Coeurjolly, D., Klette, R.: Digital planarity-A review. Discrete Appl. Math. 155, 468-495 (2007)

[3] Bülow, T., Klette, R.: Digital curves in 3D space and a linear-time length estimation algorithm. IEEE TPAMI 24, 962-970 (2002)

[4] Chen, J., Han, Y.: Shortest paths on a polyhedron. In: Proc. SoCG, pp. 360-369 (1990)

[5] Coeurjolly, D., Miguet, S., Tougne, L.: 2D and 3D visibility in discrete geometry: An application to discrete geodesic paths. PRL 25, 561-570 (2004)

[6] Cohen-Or, D., Kaufman, A.: Fundamentals of surface voxelization. GMIP 57, 453$461(1995)$

[7] Coxeter, H.S.M.: Regular Polytopes. Dover Pub. (1973)

[8] Kimmel, R., Sethian, J.A.: Computing geodesic paths on manifolds. Proc. Natl. Acad. Sci. USA, 8431-8435 (1998)

[9] Klette, R., Rosenfeld, A.: Digital Geometry: Geometric Methods for Digital Picture Analysis. Morgan Kaufmann, San Francisco (2004)

[10] Li, F., Klette, R.: Analysis of the rubberband algorithm. Image Vision Comput. 25, 1588-1598 (2007)

[11] Martínez, D., Velho, L., Carvalho, P.C.: Computing geodesics on triangular meshes. Computers \& Graphics 29, 667-675 (2005)

[12] Mitchell, J.S.B., Mount, D.M., Papadimitriou, C.H.: The discrete geodesic problem. SIAM J. Comput. 16, 647-668 (1987)

[13] Polthier, K., Schmies, M.: Straightest geodesics on polyhedral surfaces. In: ACM SIGGRAPH 2006 Courses, pp. 30-38 (2006)

[14] Surazhsky, V., Surazhsky, T., Kirsanov, D., Gortler, S.J., Hoppe, H.: Fast exact and approximate geodesics on meshes. ACM TOG 24, 553-560 (2005)

[15] Toutant, J.L., Andres, E., Roussillon, T.: Digital circles, spheres and hyperspheres: From morphological models to analytical characterizations and topological properties. Discrete Appl. Math. 161, 2662-2677 (2013)

[16] Xin, S.Q., Wang, G.J.: Improving Chen and Han's algorithm on the discrete geodesic problem. ACM TOG 28, Art. 104 (2009) 
[17] Xin, S.Q., Ying, X., He, Y.: Constant-time all-pairs geodesic distance query on triangle meshes. In: Proc. I3D 2012, pp. 31-38 (2012)

[18] Ying, X., Wang, X., He, Y.: Saddle vertex graph (SVG): A novel solution to the discrete geodesic problem. ACM TOG 32, Art. 170 (2013)

[19] Ying, X., Xin, S.Q., He, Y.: Parallel Chen-Han (PCH) algorithm for discrete geodesics. ACM TOG 33, Art. 9 (2014)

\section{Appendix}

Table. C-octants and Q-octants

\begin{tabular}{|c|c|c|}
\hline C-oct & Q-octants & Notation \\
\hline \hline $\mathbb{C}_{1}$ & $\mathbb{Q}_{1}, \ldots, \mathbb{Q}_{6}$ & +++ \\
\hline $\mathbb{C}_{2}$ & $\mathbb{Q}_{7}, \ldots, \mathbb{Q}_{12}$ & -++ \\
\hline $\mathbb{C}_{3}$ & $\mathbb{Q}_{13}, \ldots, \mathbb{Q}_{18}$ & +-+ \\
\hline $\mathbb{C}_{4}$ & $\mathbb{Q}_{19}, \ldots, \mathbb{Q}_{24}$ & --+ \\
\hline $\mathbb{C}_{5}$ & $\mathbb{Q}_{25}, \ldots, \mathbb{Q}_{30}$ & ++- \\
\hline $\mathbb{C}_{6}$ & $\mathbb{Q}_{31}, \ldots, \mathbb{Q}_{36}$ & -+- \\
\hline $\mathbb{C}_{7}$ & $\mathbb{Q}_{37}, \ldots, \mathbb{Q}_{42}$ & +-- \\
\hline $\mathbb{C}_{8}$ & $\mathbb{Q}_{43}, \ldots, \mathbb{Q}_{48}$ & --- \\
\hline
\end{tabular}

\begin{tabular}{|l|l|}
\hline Q-oct & Notation \\
\hline $\mathbb{Q}_{1}$ & $(+x,+y,+z)$ \\
\hline $\mathbb{Q}_{7}$ & $(-x,+y,+z)$ \\
\hline $\mathbb{Q}_{13}$ & $(+x,-y,+z)$ \\
\hline $\mathbb{Q}_{19}$ & $(-x,-y,+z)$ \\
\hline $\mathbb{Q}_{25}$ & $(+x,+y,-z)$ \\
\hline $\mathbb{Q}_{31}$ & $(-x,+y,-z)$ \\
\hline $\mathbb{Q}_{37}$ & $(+x,-y,-z)$ \\
\hline $\mathbb{Q}_{43}$ & $(-x,-y,-z)$ \\
\hline
\end{tabular}

\begin{tabular}{|l|l|}
\hline Q-oct & Notation \\
\hline $\mathbb{Q}_{2}$ & $(+y,+x,+z)$ \\
\hline $\mathbb{Q}_{8}$ & $(+y,-x,+z)$ \\
\hline $\mathbb{Q}_{14}$ & $(-y,+x,+z)$ \\
\hline $\mathbb{Q}_{20}$ & $(-y,-x,+z)$ \\
\hline $\mathbb{Q}_{26}$ & $(+y,+x,-z)$ \\
\hline $\mathbb{Q}_{32}$ & $(+y,-x,-z)$ \\
\hline $\mathbb{Q}_{38}$ & $(-y,+x,-z)$ \\
\hline $\mathbb{Q}_{44}$ & $(-y,-x,-z)$ \\
\hline
\end{tabular}

\begin{tabular}{|l|l|}
\hline Q-oct & Notation \\
\hline $\mathbb{Q}_{3}$ & $(+y,+z,+x)$ \\
\hline $\mathbb{Q}_{9}$ & $(+y,+z,-x)$ \\
\hline $\mathbb{Q}_{15}$ & $(-y,+z,+x)$ \\
\hline $\mathbb{Q}_{21}$ & $(-y,+z,-x)$ \\
\hline $\mathbb{Q}_{27}$ & $(+y,-z,+x)$ \\
\hline $\mathbb{Q}_{33}$ & $(+y,-z,-x)$ \\
\hline $\mathbb{Q}_{39}$ & $(-y,-z,+x)$ \\
\hline $\mathbb{Q}_{45}$ & $(-y,-z,-x)$ \\
\hline
\end{tabular}

\begin{tabular}{|l|l|}
\hline Q-oct & Notation \\
\hline \hline $\mathbb{Q}_{4}$ & $(+z,+y,+x)$ \\
\hline $\mathbb{Q}_{10}$ & $(+z,+y,-x)$ \\
\hline $\mathbb{Q}_{16}$ & $(+z,-y,+x)$ \\
\hline $\mathbb{Q}_{22}$ & $(+z,-y,-x)$ \\
\hline $\mathbb{Q}_{28}$ & $(-z,+y,+x)$ \\
\hline $\mathbb{Q}_{34}$ & $(-z,+y,-x)$ \\
\hline $\mathbb{Q}_{40}$ & $(-z,-y,+x)$ \\
\hline $\mathbb{Q}_{46}$ & $(-z,-y,-x)$ \\
\hline
\end{tabular}

\begin{tabular}{|l|l|}
\hline Q-oct & Notation \\
\hline \hline $\mathbb{Q}_{5}$ & $(+z,+x,+y)$ \\
\hline $\mathbb{Q}_{11}$ & $(+z,-x,+y)$ \\
\hline $\mathbb{Q}_{17}$ & $(+z,+x,-y)$ \\
\hline $\mathbb{Q}_{23}$ & $(+z,-x,-y)$ \\
\hline $\mathbb{Q}_{29}$ & $(-z,+x,+y)$ \\
\hline $\mathbb{Q}_{35}$ & $(-z,-x,+y)$ \\
\hline $\mathbb{Q}_{41}$ & $(-z,+x,-y)$ \\
\hline $\mathbb{Q}_{47}$ & $(-z,-x,-y)$ \\
\hline
\end{tabular}

Q-oct|Notation

\begin{tabular}{|l|l|}
\hline $\mathbb{Q}_{6}$ & $(+x,+z,+y)$ \\
\hline
\end{tabular}

\begin{tabular}{l|l}
\hline $\mathbb{Q}_{12}$ & $(-x,+z,+y)$ \\
\hline
\end{tabular}

$\mathbb{Q}_{18} \quad(+x,+z,-y)$

$\mathbb{Q}_{24}(-x,+z,-y)$

$\mathbb{Q}_{30}(+x,-z,+y)$

$\mathbb{Q}_{36}(-x,-z,+y)$

\begin{tabular}{|l|l|}
\hline $\mathbb{Q}_{42}$ & $(+x,-z,-y)$ \\
\hline
\end{tabular}

\begin{tabular}{|l|l|}
\hline $\mathbb{Q}_{48}$ & $(-x,-z,-y)$ \\
\hline
\end{tabular} 
Table. Summary of results

\begin{tabular}{|r|l|l|l|l|r|r|r|}
\hline$r$ & $s$ and its q-octant & \multicolumn{2}{|c|}{$t$ and its q-octant } & $\boldsymbol{\pi}_{r}^{\mathbb{Z}}(s, t)$ & $d_{i, j}^{(48)}$ & Time $(\mu s)$ \\
\hline \hline 10 & $(0,3,10)$ & $\mathbb{Q}_{1}$ & $(4,6,7)$ & $\mathbb{Q}_{1}$ & 7 & 0 & 53 \\
10 & $(4,-4,8)$ & $\mathbb{Q}_{13}$ & $(2,-9,4)$ & $\mathbb{Q}_{18}$ & 8 & 1 & 61 \\
10 & $(7,1,7)$ & $\mathbb{Q}_{2}$ & $(-3,7,-6)$ & $\mathbb{Q}_{36}$ & 21 & 6 & 81 \\
20 & $(1,5,19)$ & $\mathbb{Q}_{1}$ & $(11,12,12)$ & $\mathbb{Q}_{1}$ & 16 & 0 & 111 \\
20 & $(-4,10,17)$ & $\mathbb{Q}_{7}$ & $(-20,3,2)$ & $\mathbb{Q}_{10}$ & 24 & 3 & 145 \\
20 & $(-7,3,-18)$ & $\mathbb{Q}_{32}$ & $(4,-10,17)$ & $\mathbb{Q}_{13}$ & 52 & 8 & 330 \\
50 & $(0,-12,49)$ & $\mathbb{Q}_{13}$ & $(8,-18,46)$ & $\mathbb{Q}_{13}$ & 11 & 0 & 84 \\
50 & $(30,1,40)$ & $\mathbb{Q}_{2}$ & $(46,18,8)$ & $\mathbb{Q}_{4}$ & 40 & 2 & 261 \\
50 & $(35,-35,-4)$ & $\mathbb{Q}_{41}$ & $(-12,-13,47)$ & $\mathbb{Q}_{19}$ & 81 & 4 & 445 \\
100 & $(24,61,-76)$ & $\mathbb{Q}_{25}$ & $(57,58,-58)$ & $\mathbb{Q}_{25}$ & 34 & 0 & 167 \\
100 & $(-39,-48,-79)$ & $\mathbb{Q}_{43}$ & $(-88,-17,-45)$ & $\mathbb{Q}_{45}$ & 66 & 2 & 315 \\
100 & $(-11,78,61)$ & $\mathbb{Q}_{12}$ & $(98,-17,7)$ & $\mathbb{Q}_{16}$ & 170 & 6 & 1000 \\
200 & $(116,115,115)$ & $\mathbb{Q}_{3}$ & $(176,62,73)$ & $\mathbb{Q}_{3}$ & 93 & 0 & 392 \\
200 & $(33,33,194)$ & $\mathbb{Q}_{1}$ & $(199,14,11)$ & $\mathbb{Q}_{4}$ & 242 & 3 & 1292 \\
200 & $(46,161,110)$ & $\mathbb{Q}_{6}$ & $(-87,-2,180)$ & $\mathbb{Q}_{20}$ & 230 & 4 & 1677 \\
500 & $(-13,406,291)$ & $\mathbb{Q}_{12}$ & $(-250,340,268)$ & $\mathbb{Q}_{12}$ & 239 & 0 & 1178 \\
500 & $(50,-494,58)$ & $\mathbb{Q}_{18}$ & $(171,-226,412)$ & $\mathbb{Q}_{13}$ & 439 & 1 & 2925 \\
500 & $(-31,433,248)$ & $\mathbb{Q}_{12}$ & $(117,-171,-455)$ & $\mathbb{Q}_{37}$ & 1142 & 8 & 33347 \\
1000 & $(25,-929,368)$ & $\mathbb{Q}_{18}$ & $(539,-637,551)$ & $\mathbb{Q}_{18}$ & 628 & 0 & 5159 \\
1000 & $(384,917,-104)$ & $\mathbb{Q}_{29}$ & $(110,504,-857)$ & $\mathbb{Q}_{25}$ & 892 & 2 & 7771 \\
1000 & $(932,300,-204)$ & $\mathbb{Q}_{28}$ & $(-637,705,311)$ & $\mathbb{Q}_{11}$ & 1889 & 5 & 61852 \\
\hline
\end{tabular}

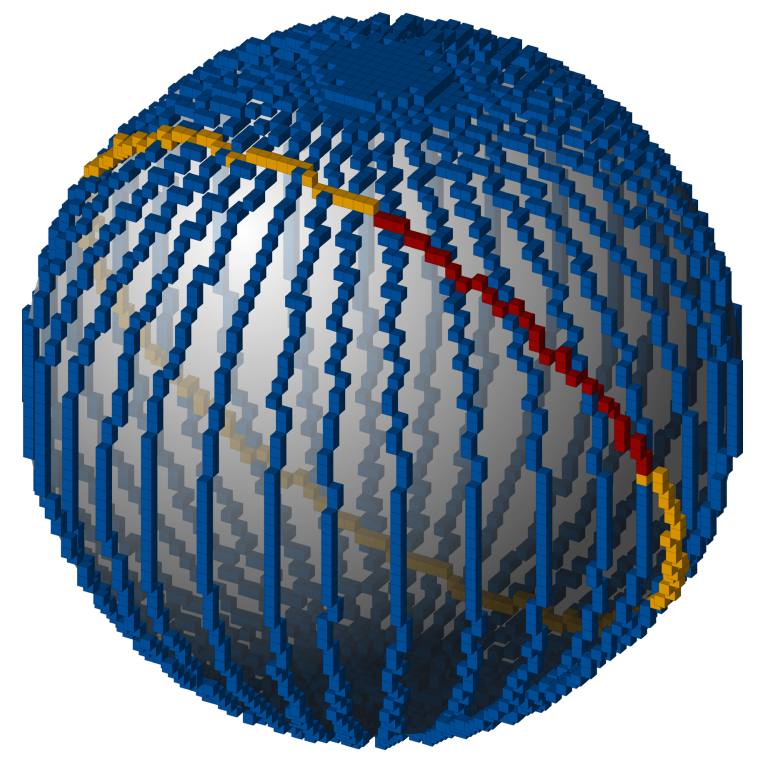

Figure. Discrete spherical geodesics and their corresponding circles for $r=30$. The sequence of red voxels is $\boldsymbol{\pi}_{r}^{\mathbb{Z}}(s, t)$ with $s(8,25,14) \in \mathbb{Q}_{6}$ and $t(29,3,6) \in \mathbb{Q}_{3}$, which, when combined with $\boldsymbol{\pi}_{r}^{\mathbb{Z}}(t, s)$, shown in yellow, yields the discrete 3D geodesic circle passing through $s, t$, and centered at $o$. Shown in blue are 16 longitude circles produced by extending the geodesics from source points taken from the discrete great circle on $z x$-plane to destination point $t(0,30,0)$ for each. 